\title{
Resveratrol and apoptotic proteins enhance radiosensitivity
}

Perforin and granzyme B, two apoptotic proteins primarily expressed in lymphocytes, are also expressed in prostate cancer cells where they are upregulated in response to treatment with resveratrol, a stilbenoid found in grapes and peanuts. These data could explain the synergistic effect of resveratrol and radiation therapy on cell death, according to a study published in the Journal of Andrology.

Perforin and granzyme B are both required to induce apoptosis-perforin cannot induce apoptosis alone, but creates pores enabling granzyme B to enter cells and induce cell death. Although expression of these proteins is well established in activated lymphoid effector cells and peritumoral expression has been linked with prognosis in some cancers, expression has not previously been shown in prostate cancer cells.

The team from the University of Missouri School of Medicine used a combination of RT-PCR, immunohistochemistry and Western blot to show that both perforin and granzyme $\mathrm{B}$ are constitutively expressed in PC3 and DU145 prostate cancer cells. When they investigated the effect of treating these cells with resveratrol at concentrations ranging from 0 to $50 \mu \mathrm{M}$ followed by radiation treatment, the investigators found that the cells were resistant to radiation treatment unless it was preceded by resveratrol-in other words, resveratrol enhanced the effect of radiation therapy.

Interestingly, RT-PCR experiments revealed that the expression of perforin and granzyme B mRNA was increased in cells treated with resveratrol. Additionally, expression of perforin and granzyme $B$ in the cell lines was further increased in cells treated with both resveratrol and radiation, suggesting that the enhanced apoptotic effect of resveratrol and radiation therapy could be due to an increase in these proteins.

The authors have also reported constitutive expression of granzyme B in melanoma cell lines and shown that it is upregulated by resveratrol treatment, raising the possibility that this mechanism is not specific to a particular cell line or type of tumor and that it could be exploited in the development of new cancer therapies.

\section{Annette Fenner}

Original article Fang, Y. et al. A possible role for perforin and granzyme $B$ in resveratrol enhanced sensitivity of prostate cancer. J. Androl. doi:10.2164/ jandrol.111.015164

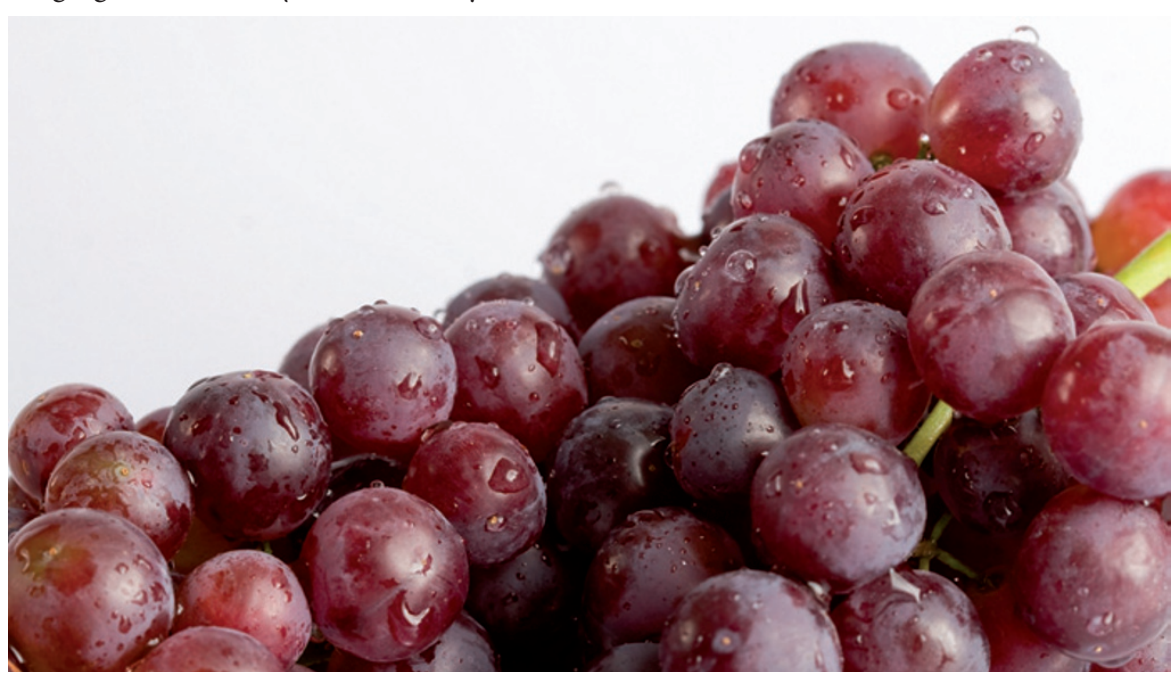

